\title{
34. COMMISSION DE LA MATIERE INTERSTELLAIRE ET DES NEBULEUSES PLANETAIRES
}

Président: M. H. C. van de Hulst.

Membres: MM. Adams, L. H. Aller, Ambartsumian, Baade, Beals, Bertaud, Bok, Bowen, Dufay, D. S. Evans, Fesenkov, Greenstein, Gurzadian, J. S. Hall, Haro, Hatanaka, Herbig, Hiltner, Kharadze, Lambrecht, Malmquist, Menzel, P. W. Merrill, Minkowski, G. Münch, Öhman, Oort, Parenago, Rozhkovski, Schalén, Schoenberg, Shajn†, Sobolev, Spitzer, Stebbins, Strömgren, Struve, Swings, ter Haar, Thackeray, Trumpler†, Vashakidze $\dagger$, Velghe, Vorontsov-Velyaminov, Whipple, Whitford, O. C. Wilson, W. H. Wright, Zanstra.

\section{4a. Sous-commission pour la Préparation d'un Catalogue des} NÉBuleuses Galactiques

Président: M. G. A. Shajn†.

Membres: MM. Bok, Thackeray.

The task of writing a report for an I.A.U. commission is made difficult for the very same reason why it is worth while at all: the growth of astronomy. Some subjects remain stationary while others, initially small, may gain enormous importance in the course of a few years. For that reason continual doubts about the borderlines of the commissions exist and an even greater turnover of membership than is actually in effect by means of the nomination and retiring procedure might be advisable.

Commission 34 on Interstellar Matter was established after the General Assembly of r 938 as a small commission of seventeen members. In 1946 the Galactic Nebulae, formerly under Commission 28 , were added to its field. The combination remains, to some extent, arbitrary. For instance, at present the subject of interstellar matter is strongly interwoven with that of diffuse nebulae, but has no closer relation to planetary nebulae than it has, for example, to galactic structure, to spectral photometry, to stellar evolution and to radio astronomy.

For the present report I have to thank thirty-six of the fifty commission members who sent me their reports or notes about recent work and in addition a number of nonmembers who supplied very useful information. Our thanks are also due to those members who have served on this Commission for many years and have now expressed the desire to be replaced by younger men.

\section{REviEWS}

The rapid expansion of the subject had made the need for summarizing articles or books more acute. Fortunately, a fair number of these have appeared in the last years or are in the course of preparation.

A semi-popular book by Dufay, Nébuleuses galactiques et matière interstellaire, appeared in 1953. Safronov( $\mathrm{I}$ ) and Spitzer (2) reviewed the present knowledge of interstellar matter, while Spitzer also prepared a mimeographed set of lecture notes. Many will welcome also Aller's Astrophysics II: Nuclear transformations, stellar interiors, and nebulae (1954), which contains a great deal about our subject. A monograph on interstellar matter by Lambrecht will appear in the spring of I955. In the field of gaseous nebulae we have the German edition of Vorontsov-Velyaminov's book (I953) and a forthcoming book by Aller. The problem of the kinematics and dynamics of planetary nebulae will be discussed in two monographs being prepared by Minkowski and Wilson and by Gurzadian. Finally, we may note that two important symposium reports will appear in the spring of 1955, Gas Dynamics of Interstellar Clouds, the report of the second 
symposium on this subject, organized by I.A.U. and I.U.T.A.M. at Cambridge, July I953, and the report of the Liège Symposium of July I954 on 'Dust in Astronomical Objects'.

\section{General SURVEY}

While it is impossible to give a critical review, or even a fairly complete enumeration, of the work done in the past three years, it is possible to indicate the general trends and to mention some of the highlights. The same types of ideas spring up at different places, either independently or fostered by the internationa! contacts. At any rate, in spite of the fact that most active research workers find it impossible to keep accurately informed about what the others are doing, there is not a divergence between the lines of research pursued at different countries but a happy convergence. The most noticeable difference is that the countries that do not possess the most powerful instruments are given somewhat more to speculation.

If it would be necessary to characterize the present trends in a few lines, the shifts of emphasis in the last years might be described as follows.

In studies of interstellar matter on a galactic scale the emphasis has shifted from the mapping of dark clouds to the mapping of emission regions and of the O-associations to which they belong. No study reaching beyond rooo ps, say, should be complete without reference to the existence of spiral arms.

On a far larger scale, up to the galactic centre and beyond, the $2 \mathrm{I} \mathrm{cm}$. observations are giving strikingly new results. As there are no other direct means to investigate interstellar matter at those distances, this subject will not be included in this report. (See Commissions 33 and 40.)

In spite of a great deal of attention, no major advances have been made in the interpretation of interstellar reddening and interstellar polarization.

In the observational study of interstellar gas clouds there has been a shift of the attention from the absorption lines to the study of emission regions and diffuse nebulae. Yet the absorption lines continue to give very important information.

Ionization problems in the theory of the interstellar gas have been replaced by dynamical problems as the central topic. A working hypothesis about the origin of interstellar clouds has been reached and the importance of magnetic fields is no longer doubted.

The problems of the relation between stars and clouds continue to receive a lot of attention, mainly with a view to problems of stellar evolution, but still present many unsolved problems.

Striking progress in the study of planetary nebulae has been made in the improvement of observational techniques, giving better photometric, spectrophotometric and kinematic data than were available before. The main emphasis in theoretical studies is shifting to problems of dynamics.

After this attempt at a general characterization, which is necessarily incomplete, detailed comments on each of these topics will follow.

\section{Distribution of Interstellar Matter on a Galactic Scale}

The old aim of drawing a map of the distribution of interstellar gas and solid particles in our galaxy still remains a prime object of many investigations. It is convenient to make a distinction between the region of the galactic plane farther than $\mathrm{I}$ kps from the Sun and the region closer to the Sun.

Studies in the region with $r>$ I kps. may be held to fall primarily under Commission 33 on Galactic Structure. The report of the first I.A.U. Symposium, on 'Co-ordination of Galactic Research', should be required reading for anyone embarking on a programme in that region. The studies of the $2 \mathrm{I} \mathrm{cm}$. line in Holland and elsewhere and of the Oassociations and associated nebulae by Morgan and co-workers, that have enabled us to draw a first rough map of the spiral arms in our galaxy, are well known. Also Gaze (3) 
has plotted three spiral arms from I5o nebulae and finds general agreement with Morgan's work.

The large-scale arrangement of ionized hydrogen clouds and neutral hydrogen clouds in the spiral arms is similar. Recent measurements of the small deviations from the galactic plane tend to confirm this. Also the neutral hydrogen clouds and the regions of obscuration are found to have a good overall correlation, as shown by the studies of Bok and his co-workers (4) at intermediate and high latitudes.

Important new data are obtained by the study of multiple absorption lines. The continuation of Münch's (5) survey of interstellar lines in distant stars has revealed a striking variation of the velocity distribution of the clouds in different spiral arms and from place to place in the same spiral arm. The great width and large doublet ratios of the $\mathrm{CaII}$ or $\mathrm{Na}$ lines produced by the Perseus arm, on stars of the double cluster for example, indicate a velocity dispersion at least twice as large as that shown by the lines formed in the Orion arm.

These observations seem to confirm the suspicion expressed by van de Hulst, Muller and Oort (6) that the elongated features, pointing towards the Sun, of the distribution of interstellar atomic hydrogen are spurious and have been caused by variations of the velocity distribution of the clouds from the values determined from the nearby stars in Adams' list. In the present phase of the $21 \mathrm{~cm}$. observations, in which no individual clouds can be detected, this information is extremely important. In a later phase the $2 I \mathrm{~cm}$. observations made with bigger instruments may permit a similar distinction of individual clouds and their motions.

Münch has also continued his search for interstellar clouds at high galactic latitudes. Apart from those already described ( () , a star HD 215733 at $l=54^{\circ}, b=37^{\circ}, r=2400 \mathrm{ps}$. has now been found to show four well-separated interstellar lines. The occurrence of gas clouds as far as Iooo ps. from the plane raises interesting questions regarding their life and stability. Additional information on the $2 \mathrm{I} \mathrm{cm}$. radiation in these fields is being obtained.

Studies in the region with $r<\mathrm{I}$ kps. have a somewhat different character. The $\mathrm{O}$ associations are fairly few and the $2 \mathrm{I} \mathrm{cm}$. observations do not admit of a very clear interpretation. Here the interstellar absorption lines and the study of dark regions are quite useful; for an earlier survey of this region see Binnendijk (8).

Beals and $\mathrm{Oke}(9)$ have published a table of the intensities of the $\mathrm{K}$ and $\mathrm{D}$ lines of I82 stars, based on over Iooo spectra. The best distance-intensity relations are

$$
r=35 K=3 I D \text {; }
$$

these relations rarely give an individual distance in error by more than $25 \%$.

Although heavily obscured stars are sometimes observed at very large distances, of the order of several kps., the ordinary method of dark cloud investigations is limited to smaller distances. A hundred square degree area in Taurus has been investigated by Adolfsson (ro) down to the twelfth magnitude. Three dark nebulae at distances 30-70, I75 and 600 parsec are found. Eklöf (rI) has made a similar investigation in a region in Auriga. The ratio of total to selective absorption is found the same in these fields. Velghe (12) is making an investigation of a dark region in Cygnus, consisting of the determination of monochromatic magnitudes at three wave-lengths and of spectral types. A similar study of the southern fields in Vela and Crux-Centaurus, for which plates will be obtained at Bloemfontein, is in preparation.

A large amount of statistical work on interstellar absorption at the Abastumani Observatory has resulted in three major papers: a catalogue of colour indices of 14,000 stars by Kharadze (13); a catalogue of photo-electric colours of B8 and B9 stars by Nikonov (14); and a general discussion of galactic absorption, based on colour excesses of extragalactic nebulae and long-period variables and other methods, by Vashakidze (xs). Later work includes two determinations of the plane of symmetry of dark matter from colour indices by Torondzhadze and Kotchlashvili (16), and from a catalogue of 797 dark nebulae by Havtasi. Meanwhile, further colour excesses in the Kapteyn areas $\delta=30^{\circ}$ 
are being determined by Kharadze and roo colour excesses of short-period variables have been obtained by Alanya.

The possibility of gauging the other spiral arms and even the distant parts of the galaxy should not lead to a neglect of the parts close to the Sun. Techniques have been developed, for instance, to measure photo-electrically weaker interstellar lines and weaker degrees of polarization than the now usual limits. The hope may be expressed that suitable programmes will be started for mapping the nearest regions.

A few investigations on interstellar matter in extragalactic nebulae may be reported here (for fuller references see Commission 28). At the Crimean Observatory integrated $\mathrm{H} \alpha$ brightnesses and masses were found for objects in $\mathrm{M} 33, \mathrm{M}$ ror, and NGC 6822; they contain of the order of $10^{4}$ solar masses. Over eighty emission nebulae were catalogued in M 33 from filter photographs. The relation to spiral arms was discussed.

Nebulae and interstellar matter in the Magellanic Clouds are being investigated at Pretoria. The studies refer to: the S-shape of many nebulae in the Clouds (ry); the nebula of 30 Doradus and the stars involved (Feast); detection of interstellar $\mathrm{Ca}^{+}$lines in the Large Cloud (Feast (18)); and a search for obscuration in the Small Cloud by counts of extragalactic nebulae (Wesselink). Thackeray notes a confusion of names that has occurred in the literature: 30 Doradus (centre of NGC 2070) is not the same as $S$ Doradus (in a nearby unnamed nebula).

The presence of a measurable extinction in intergalactic space is still uncertain. This subject is much more closely related to Commission 28 on Extragalactic Nebulae than with Commission 34; for a brief review see Whitford ( $x$ g).

\section{Reflexion Nebulae and Small Dark Clouds}

Theoretical investigations of reflection nebulae have been continued by Schalén(20). Kaplan and Klimishin (2x) have applied Ambartsumian's theory of diffuse radiation to reflexion nebulae. Fesenkov and Rozhkovski have made photometric studies of reflexion nebulae together with theoretical computations resulting in an estimate of their masses. A large programme of absolute photometry of the Milky Way by Kariagina is in progress.

A subject of great importance is the physical and evolutionary status of the globules. Rozhkovski (22) concluded from a statistical study that their connexion with large emission nebulosities is not accidental. This finding is in keeping with the evidence presented and the theories developed at the Cambridge Symposium, but contradicts a suggestion by Martynov (23) who, impressed by the fact that rich star fields show numerous thin filaments of dark matter of density $\mathrm{IO}^{-23}$ to $\mathrm{IO}^{-22}$, suggests that such filaments seen along their length are observed as globules.

Schoenberg (24) has continued his studies of dark nebulae. A new method now applied at the Wendelstein Observatory is based on photographic photometry of the background between the stars. The further reduction is as usual. A catalogue, which will contain positions, diameters and estimated absorption values of I200-1500 small dark clouds is in preparation. It is possible to distinguish several distinct types of dark clouds.

Thackeray (25) has made a further study of the remarkably small dark clouds in the region of IC 2944. The smallest cloud detected has a size of the order of rooo astronomical units. Apparent obscuration of a star at the edge of one cloud was found. Wachmann (26) suggests that also FU Orionis has been occulted by a small dark cloud.

\section{Extinction and Polarization; Properties of Grains}

The most important clue to the nature of the interstellar grains is still the law by which the interstellar extinction depends on the wave-length.

An important study of the interstellar extinction law has been completed by Miss Divan (27). On the basis of photographic spectral photometry of $O$ and $B$ stars from 6100-3130 $\AA$ she finds precisely the same law of reddening for many stars in different 
regions of the sky, including the Orion region. The variation of the three intrinsic parameters (Balmer jump, and the two gradients at either side) with spectral type and luminosity was studied with care. It is concluded that no unknown differences due to intrinsic differences of reddened and unreddened stars remain to vitiate the extinction law. This precaution seems to answer the objection raised by Melnikov (28), who computed that the wings of the Lyman $\alpha$ line in B and early A stars are wide enough to reach into the violet region and must depend on absolute magnitude.

It thus appears that no certain deviations from 'the normal law of reddening' have been found anywhere, a fact that presents a major theoretical problem if the ideas about a continual growth and destruction of grains are correct. Whitford holds that the photoelectric scanning spectrograph may reach a still greater precision. Further observations of the extinction law have been made by van Rhijn (29), Borgman (30) and Kienle (3r). This subject deserves continuing attention.

Idliss (32) claims that the size distribution of interstellar grains that corresponds to the dependence of interstellar absorption on wave-length is a $\rho^{-4}$ law and that this law agrees with the one expected from fragmentation of solid bodies. Zirin (33) suggests that fragmentation provides the nuclei for the growth of new particles. In this connexion it may be recalled that the total amount of meteoritic dust shed into interstellar space by all planetary systems like ours, by the processes considered in Oort's theory on the origin of the comets, may not be negligible.

While it would seem that there are certainly no urgent observational reasons to abandon the idea of an ice-like composition of the grains (34), various rather deviating suggestions have recently been made. The possible growth of electrically polarized ice needles in interstellar space was described by Kahn (35).

In a discussion of the physical nature of the grains, as may be deduced from their scattering and extinction, Güttler (36) arrives at the conclusion that metallic particles will do better. In a later paper he derives formulae and numerical results for extinction of light by a dielectric sphere with an absorbing spherical nucleus. Güttler now studies the theoretical problem of the origin of the interstellar grains.

Even more drastic is the suggestion by Schatzman and Cayrel (37) that the interstellar particles might consist of graphite. This suggestion was accompanied by laboratory investigations of the polarization produced by oriented graphite flakes. Schatzman calls the attention of commission members to the possibility of an observational test by observing the wide absorption band of graphite near $3000 \AA$.

One of the crucial observational data on the composition of the grains is the diffuse light in the galaxy. It may be good to draw the attention of members of Commissions 22 and 34 to the need of new observations.

The study of interstellar polarization is continued with great activity. Most authors emphasize the importance of the polarization studies in connexion with galactic structure. The ultimate goal of interstellar polarization studies should be to trace the magnetic fields of our galaxy and to see if Chandrasekhar's and Fermi's (38) suggestion of fields stretched along the spiral arms is right.

Hoag (39) finds from polarization measurements in five open clusters that the ratio of polarization to reddening is a constant for each cluster, which varies in such a way with galactic longitude as to indicate that the grains are aligned with their short axes along the spiral arm. Lodén is using the quadruple image polarigraph in order to measure polarization in two colours in selected open clusters. At the Liège Symposium new observations on polarization were presented by Blamont and Courtès, by Behr, and by Hall. The last-mentioned showed evidence for predominant alignment of particles perpendicular to the filaments in the Pleiades nebulae. Hall has also completed the measurements of the polarization of the 1332 B star in the Washburn catalogue. Hiltner's observations have included magnitudes in the three-colour VBU system, polarization and slit spectra (for spectral type) all made at the McDonald Observatory. Some partial results have been published (40). A full list of these data for over Iooo stars will appear before the Dublin meeting. 
Sharpless is determining photographically with a calcite filter and the 40-inch reflector of the U.S. Naval Observatory the polarization of a number of bright objects in M 33 in order to investigate the large-scale structure of the magnetic field of the galaxy. Mrs Elvius (41) has studied the polarization in dark clouds in a number of extragalactic nebulae. There is good evidence in the case of NGC 5055 that the electric vector follows the spiral arms, as might be expected on the basis of the theory of Greenstein and Davis.

At Harvard Observatory Mrs Elske Smith is completing the reduction of measurements of several hundred southern stars.

The polarization in emission nebulae has a mixed origin. Its interpretation is connected with that of the continuous spectrum that has three components, electron scattering, dust scattering, free-free emission, that are difficult to separate, as appears from the communications by Shajn(42), by Mrs Martel (43) and by Seaton (44) at the Liège Symposium. The radial polarization (magnetic vector pointing to central stars) of the Orion nebula is certainly due to scattering by free electrons. Rozhkovski (45) reports that it is preserved up to its remotest parts. On the other hand, he concludes that the polarization in M 8 and M 20 is of an interstellar character. Work on the dust region of M 8 is continued in collaboration with Matiagin. Walraven has found a high value for the polarization of the Crab Nebula.

L. Davis (Pasadena) has summarized on several occasions (46) the theories of interstellar polarization. He concludes that Gold's (47) mechanism based on a relative velocity of gas and dust is inacceptable. The orienting mechanism with paramagnetic particles spinning in a magnetic field of the order of $10^{-5}$ gauss that is directed along a spiral arm is preferred. In another paper Davis ${ }^{(48)}$ has tried to predict the asymmetry of cosmicray flux on the basis of a thus directed field. The hypothesis finds some support in the observational data. A further discussion of the interstellar polarization mechanism was given by Burbidge (49), who concludes that Davis' and Greenstein's mechanism is barely sufficient, if the field of Chandrasekhar and Fermi is supposed.

It may be emphasized that these theories are all based on the Gans theory (dimensions $\ll \lambda)$. Theuse of this theory may give some insight, but will predict too high a valne for the ratio of polarization to absorption, and the colour dependence cannot be expected to follow from the Gans theory at all. Here is an obvious need for a more precise optical theory or, perhaps, laboratory experiments. It should also be emphasized that apart from the large-scale programmes on polarization discussed above (and suggested for the nearer regions on p. 492) a few suitable stars should be studied with the utmost precision in the widest wave-length range possible. So far it is only known that the polarization in the red is about $80 \%$ of the polarization in the blue. Also the presence of a component of elliptical polarization should be tested with great care.

\section{IONIZATION AND TEMPERATURE OF THE INTERSTELLAR GAS}

The interstellar radiation field has been newly computed by Lambrecht and Zimmermann (50), thus correcting the work of Dunham of 15 years ago. The variation of interstellar absorption with wave-length and the still uncertain deviations of stars from black bodies have been included. New computations for interesting volumes in the galactic system are planned. The ionization functions ' $N_{2} N_{e} / N_{1}$ have been determined for the most important atoms and ions using the new radiation field. On this basis the extent of the $\mathrm{H}$ II regions and the abundance ratio $\mathrm{Na} / \mathrm{Ca}$ have been newly discussed (5r). One uncertain point forms the diffuse radiation, i.e. the radiation scattered by the grains, that was not included in these computations.

Another study of the radiation field in the continuous spectrum and in Lyman $\alpha$ was made by Kaplan(52). Kaplan and Gopasiuk (53) computed the interstellar hydrogen emission arising from collisional excitation by electrons.

Herzberg (54) has reviewed at the Liège Symposium the identification of the interstellar lines. Two sharp lines at $3934^{.29}$ and $3579^{\circ} 04 \AA$ still remained unidentified. Herzberg emphasizes the importance of attempts to find further interstellar lines, particularly at 
the ultra-violet end of the accessible spectrum. Herzberg further suggests that the diffuse interstellar lines may be due to diffuse (pre-dissociated) absorption bands of triatomic (or polyatomic) free radicals, similar to $\mathrm{HCO}$.

Further points of interest about the interstellar molecules may be found in the Liège Symposium report. Most important of all is the $\mathrm{H}_{2}$ molecule. Some local studies of $2 \mathrm{Im}$. line radiation in Taurus showed that the regions with the strongest obscuration do not have the strongest hydrogen lines. This may be due to the formation of $\mathrm{H}_{2}$ molecules. Kahn (55) has discussed the formation and dissociation of $\mathrm{H}_{2}$ theoretically and concludes that most of the hydrogen in dense clouds may be molecular.

Another problem of basic importance is the temperature of the interstellar gas. The papers by Spitzer (56) on the temperature of interstellar matter were somewhat difficult to read because of the many details. Many will therefore welcome the reviews and numerical illustrations given of these calculations by Savedoff $(57)$ at the Cambridge Symposium and by Spitzer (58) in his Russell Lecture.

For the Hir regions there is no direct observational test. The radio data may give fuller information in the near future. At present they indicate an upper limit of the order of $20,000^{\circ}$ but the possible presence of non-thermal emission makes it seem that the computed value of $8000^{\circ}$ may just as well be correct. The widths of the interstellar absorption lines indicate an upper limit of $40,000^{\circ}$, but must certainly be interpreted by internal motions in the clouds of the order of $5 \mathrm{~km}$. $/ \mathrm{sec} .(59,60)$.

The $2 \mathrm{I} \mathrm{cm}$. results initially indicated a value near $100^{\circ}$ for the $\mathrm{HI}_{\mathrm{I}}$ regions, but more recent determinations in Holland make it $125^{\circ}$. The calibration is not final and there is some divergence with the preliminary Australian results that would correspond with a still higher temperature. These values are sufficiently far above Spitzer's computed values of $50^{\circ}$ and lower to worry about the difference. Kahn (6r) has suggested that the equilibrium is not rapidly reached and that the measured temperature is the harmonic mean of the temperatures of clouds in various stages of cooling.

\section{DyNamics OF THE INTERSTEllar Gas}

Before embarking on the more typically astrophysical problems, it should be noted that there is a vast field of specialized research in aerodynamics, magneto-hydrodynamics and plasma physics that holds a position between physics and mechanics, astrophysics and geophysics. While it is of obvious importance that members of Commission 34 should not lose track of the developments in this field, it is impossible to give an adequate review in this report. So only the more direct astrophysical applications will be mentioned.

The existence of huge clouds and diffuse nebulae has been a big problem ever since it was recognized that such formations could not keep their motion, nor continue to exist for a time comparable to the age of our galaxy. The solution has been sought in two rather different directions:

(a) Explanations with the key-word turbulence, in which the clouds have some connexion with the turbulent eddies. Gravitation and galactic rotation might be the driving factors and magnetic fields form an important element in these theories.

(b) Explanations with the key-word radiation, in which the temperature and pressure differences set up by newly born O-stars are essential. The driving power thus is derived from nuclear energy.

At the first combined symposium of I.A.U. and I.U.T.A.M. on these problems, at Paris in I949, attention was given exclusively to theories of the first type. At the second symposium, at Cambridge, r953, there was a strong switch to theories of the second type. It is quite probable now that the second idea is basically correct, but that in the elaboration of this theory certain elements of the earlier theory of interstellar turbulence will be important. It may be helpful to mention some investigations concerning theories of both types, although more than a loose juxtaposition cannot be made.

The recent theory of Oort (62) links the origin of the interstellar clouds with the origin of the expanding associations. Both the compression into clouds and the momentum of 
the clouds are thought to be consequences of the complicated dynamical effects that follow if a new-born $\mathrm{O}$-star makes a hot $\mathrm{H}$ II region inside a dense neutral cloud. One of these effects is the rocket effect (Oort and Spitzer) that can give some small clouds very high velocities. There is some evidence in the observations that the clouds move indeed away from the hot stars. (See also papers by Searle (63) and by Schlüter, Schmidt and Stumpff (64).) Münch (65), rediscussing the entire problem of the interstellar clouds with high velocities, also finds general agreement with this theory, while Shajn (66) arrives at a similar picture. He calls attention to the clustering tendency of emission nebulae and concludes that the groups are genetically connected: they form in a spiral arm, expand and gradually disintegrate.

Schatzman and Kahn (67) investigated with some simplifications the propagation of the HII border as the cloud expands and the shock wave running ahead of it in the neutral cloud. Kahn's paper (68) gives a more precise discussion of similar problems for the case of plane waves and should prove of fundamental importance. Also Kaplan (69) has studied the motion of interstellar gases taking account of the interaction with radiation.

Gurzadian finds that stars earlier than type $F$ can repel the gas in their vicinity and thus prevent accretion. In hot stars the volume in which repulsion occurs is smaller than the Hil region; he suggests that this may explain the emptiness of the central part of the Monoceros Nebula.

Finally, Frieman (7o) has suggested that the peculiar elephant trunks might be explained as due to Rayleigh-Taylor instability.

Many studies have aimed at further clarification of the problems connected with gravitational instability and with turbulence. Chandrasekhar and Fermi (7I) discuss gravitational instability in the presence of a magnetic field and the Coriolis force. Parker (72) has discussed the problems of gravitational instability and collisions between clouds. Using a new method, based on the Newtonian approximation of the Einstein equations of general relativity, McVittie (73) discusses the motion of a bounded cloud in one dimension under its own gravitation. He shows that a cloud can move as an entity held together by its self-attraction.

Fermi's(74) lecture and the two I.A.U.-I.U.T.A.M. Symposium reports give ample information on the difficult subject of turbulence and magnetic fields. Kaplan, in collaboration with Pronik, has studied the spectrum of turbulence of the interstellar gas both without (75) and with magnetic fields (76). He finds that Adams' observations can be interpreted by isotropic turbulence (77). A study of the acceleration of charged particles in a field of magnetic gaseous turbulence is also made.

Lebedinsky (78) has suggested that turbulent motions are due to the gravitational action of star clouds with masses of the order $10^{6} \mathrm{M}$, an idea that forms a remarkable counterpart to the studies of Schwarzschild and Spitzer (79), according to which the stellar velocities are influenced by massive concentrations of interstellar matter.

$\mathrm{K}$. Takakubo (80) has suggested that the turbulent motions introduced by galactic rotation form compressed regions of the size of the eddies. The compressed region may be large enough to become an $\mathrm{HI}$ region and thus to become cooler and denser, finally forming an interstellar cloud.

Ter Haar (8r) has pointed out the possibility that the dynamical factor that determines the size of interstellar gas clouds is the requirement that the motion inside them is laminar.

Finally, Pikelner (82) discusses the relation between the magnetic fields and the distribution of interstellar gas between the spiral arms. Velocities in the latter are estimated at $50-100 \mathrm{~km}$. $/ \mathrm{sec}$. and the $z$-extension at $5 \mathrm{kps}$. He suggests that this gas may be identified with the source of continuous radio emission. The bearing on theories of the origin of cosmic rays is obvious. 


\section{Diffuse Nebulae and Emission Regions}

In accordance with the resolution adopted at Rome in 1952 a sub-committee has studied the possibilities of making a general catalogue and/or atlas of emission nebulae. Data concerning systematic surveys, lists and atlases are given in the sub-committee's report. Other problems concerning diffuse nebulae should be held to fall under the competence of the full Commission 34 and, therefore, are reviewed here.

The diffuse nebulae should first of all be seen in their evolutionary significance. If the idea proposed in the preceding section under $(a)$ is correct, then the brighter diffuse nebulae should be fragments of expanding cloud complexes at an early stage of their career. This is essentially the view expressed by Oort (83) and by Shajn and Gaze (84). Discussing the relations between hot stars and diffuse nebulae the latter authors conclude that a genetic relation exists for the brighter and more compact nebulae, that often are associated with multiple stars, or with groups of hot stars. A separation of $I^{\circ}$ or more may be reached in $\mathbf{I 0}^{\mathbf{6}}$ years, so it is also probable that many more extended nebulae are now excited by hot stars that are accidentally near them. Impressive observational proof of the common occurrence of multiple stars in diffuse nebulae has been given by Sharpless (85).

Special attention was paid by Shajn and his co-workers to the structure of diffuse nebulae and its dynamical interpretation. Their conclusions may be summarized as follows:

(a) Many nebulae are filamentary with a preferential direction of the filaments. These may be real gas streams and are often aligned along the galaxy (86).

(b) Other nebulae have turbulent motion. A method for deriving the turbulent motion from brightness variations was developed and applied to the Orion nebula (87).

(c) A striking class form the nebulae with a concentration of matter at the periphery (briefly: ring-like nebulae). They are mostly very large (IO-IOO ps.) and expanding under the influence of the pressure difference of hot and cold regions and of interaction with the interstellar medium (88).

It may be commented that the Palomar Schmidt plates show a far greater complexity than might be inferred from the statement made under $(a)$ above and that the theoretical implications of the suggestion made under $(c)$ above were reviewed in the preceding section. The use of such huge rings as distance indicators of extragalactic systems was suggested by Gum and de Vaucouleurs (89).

Also Fesenkov and Rozhkovski (9o) have further studied the structure of nebulae and its relation to stars. Some forms discussed are: bright rims, chains of stars and gas knots, turbulent forms, and dark haloes around some stars.

A problem of practical importance is the ratio of gas to dust. This subject was discussed at the Cambridge Symposium. Savedoff (9r) computed the frictional force exerted upon solid grains by a stream of neutral or ionized gas and concluded that the force is so strong that dust is taken along by the interstellar gas and forms condensations wherever the gas forms condensations. Bok (92), however, made a plea for the possible existence of clouds of interstellar grains without the corresponding concentration of gas. At the Liège Symposium Bok (93) supported his point of view by evidence from the distribution of southern $\mathrm{HII}$ regions and from $2 \mathrm{I} \mathrm{cm}$. results obtained by Lilley. In studying the relation of gas and dust in emission nebulae Shajn, Gaze and Pikelner (94) find that the brighter nebulae have a continuous spectrum proportional to the $\mathrm{H} \alpha$ brightness and perhaps due to 2-photon emission. Fainter emission nebulae show in addition reflected light. All reflection nebulae may have gas up to 25 protons per $\mathrm{cm}^{3}$. The ratio of gas to dust is probably not a constant.

Although the extended but very faint emission regions have been discovered nearly twenty years ago, very few published data were available. They can be reached with narrow-band interference filters (see report of sub-committee), but even better with the more laborious process of a nebular spectrograph. Hugh M. Johnson (95) has made a spectrophotometric study of HII regions. This important investigation gives intensities 
of all measurable emission lines along 63 areas each $12^{\circ}$ long and $8^{\prime}$ wide. It is found that weak $\mathrm{H} \alpha$ emission is present near all $\mathrm{O}$ stars with $z<300$ ps. but mostly absent with $\mathrm{O}$ stars farther from the plane. The emission measures are estimated. The extent of the emitting regions is of the order of $00^{\circ}$ in most cases. By a similar method Johnson (96) also detected He emission in three diffuse nebulae. Formerly, Helium was known only in the Orion Nebula in absorption in the expected strength (97).

Among the nebulae that have received individual attention the Orion Nebula and the Cygnus Loop (NGC 6960 and 6992) hold the record, followed closely by the more prominent radio sources. The strongest galactic radio source is the Cassiopeia source. It is a major step forward that, due to the discovery and identification of this and other sources, they can be studied with optical observations. In the future this subject is likely to remain of great importance for Commission 34 just as well as for Commission 40 on radio astronomy. At present the progress is so fast in this field that it is hardly worth while to include any details of the published investigations (98).

The Orion Nebula is probably the youngest diffuse nebula we can study and altogether unique. We may just refer to the strange relation to the stars AE Aurigae and $\mu$ Colombae found by Morgan and Blaauw (99) and to the preliminary proper motions measured by Strand, that indicate an age of the order of $10^{4}$ years rather than $10^{6}$ years. Using an improved interferometer Courtès (roo) has studied the spectrum of turbulence of this and other nebulae while Shajn has tried to do the same from brightness fluctuations (see above).

Wilson and Münch have studied the variations in radial velocity, width and structure of the emission lines in the Orion Nebula. They use 3I parallel slits and photograph an area of $40^{\prime \prime}$ in a dispersion of $4.5 \AA / \mathrm{mm}$. in the light of $\mathrm{H} \alpha$, (O II) and (O III). Velocity variations of $20 \mathrm{~km}$. $/ \mathrm{sec}$. over areas as small as $5^{\prime \prime}$ are found. They conclude that the earlier data are insufficient for the study of the turbulence of the Orion Nebula.

Extensive studies of the Cygnus loop by Fesenkov, Rozhkovski and their associates have included colour and magnitude determinations of many stars in the area (ror), proper motions of the filaments (xoz) and a study of the excitation mechanism. They conciude that excitation by stellar radiation is not excluded and requires densities in the filaments of $\mathrm{IO}^{-19} \mathrm{~g} . / \mathrm{cm}^{3}$. Pikelner (103) has obtained relative intensities of the main lines and studied theoretically the temperature distribution and emission by a shock front as suggested by Oort. A single wave at velocity $I_{50} \mathrm{~km}$. $/ \mathrm{sec}$. gives a filament that is narrow enough but too faint, but two intersecting waves would give a curved filament with spectrum and brightness close to the observed characteristics.

Chamberlain (104) has computed the Balmer decrement in the presence of collisional excitation of hydrogen and applied this theory to the Cygnus loop. The result is inconclusive, both radiative and collisional excitation being possible. However, Minkowski (ro5), re-discussing the problem with new spectrophotometric data, makes a clear case for radiative excitation. In connexion with the important role of the Balmer decrement in these studies it may be of interest that M. Johnson (ro6) has measured the Balmer decrement in laboratory afterglows.

Observations of southern emission regions by direct photography and with a nebular spectrograph have been reported by Gum (ro7). Southern nebulae have also been investigated with the 74-inch telescope at Pretoria. A heavily reddened Ioth mag. $\mathrm{O}$ star was discovered in the radio source NGC 6334. Slit spectra of the central region of M 8 show its ionization to be distinctly lower than that of the Orion Nebula.

By the use of a nebular spectrograph Pikelner finds that the North America and Pelican Nebulae are excited by $\mathrm{HD}$ I99579 $\left(\mathrm{O}_{7}\right)$ and have a mass of several hundred solar masses. Photometry of nebulae with various colour filters has been reported by Wurm at the Liège Symposium. 


\section{INTERACTION OF GaS AND StaRS}

Apart from the case of $O$ stars in emission nebulae discussed above (see p. 495), several further problems fall under this head.

One is the problem to assess the relative importance of the processes of accretion, condensation and corpuscular radiation, by which gas may move from interstellar space to stars and inversely. Biermann (ro8) gave a thorough survey of this problem at the Cambridge Symposium and arrived at the tentative conclusion that most of the now interstellar gas atoms may at a time have belonged to stars. An extensive review of the same problem has also been given by Gurevich (rog).

The physical problems of accretion have been discussed by Bondi (roo), by McCrea, and by Schatzman (II) at the Cambridge Symposium. Individual problems connected with the motion of stars through gas clouds were discussed by Dodd and McCrea (Ir2).

The work of Herbig and others on T Tauri stars and early-type bright-line stars associated with nebulosities has greatly progressed. Present observational data include several hundred new discoveries on slitless spectra, II4 slit spectra, and photo-electric observations of 30 stars that show that the majority of $\mathrm{T}$ Tauri stars are variable and lie 2 or 3 magnitudes above the main sequence. A classification of stars associated with nebulosity in four groups has been given (123). (See details in the report of Commission 29.)

Uccle Observatory has started a programme of detecting bright-line stars in dark nebulae by means of a $7^{\circ}$ objective prism on the $30 \mathrm{~cm}$. astrograph. $\mathrm{H} \alpha$ emission stars associated with a dark lane in Lupus have been listed by Henize (ri4). Haro and his co-workers (Ir5) have found new T Tauri stars in Joy's Taurus-Orion field and in the region of IC 434 .

Herbig further reports that direct photographs have shown that a number of irregular variable stars (mostly early-type and with bright lines) are associated with small reflexion nebulae. Herbig (II6) and Haro (II7) independently discovered a curious type of semistellar emission nebulosities near NGC I999 and in Orion and Taurus. They have a very wide range of excitation.

No clear theoretical understanding of the widely varying phenomena exhibited by stars embedded in dense nebulae has yet been reached. This is the more intriguing as some of them might give a clue to understanding the processes by which new stars are formed from interstellar matter. A session at the Liège Symposium was devoted to these problems, with papers by Schatzman, Öpik, O. J. Schmidt, Rouskol and Ebert. Related studies have also been published by Lebedinsky (r18).

\section{Planetary Nebulae: Observational Data}

As has been customary in the past, the work on planetary nebulae shows a varied spectrum ranging from purely observational papers on photometric and spectroscopic data to highly mathematical calculations on radiative transfer and energy transformations in a gaseous shell.

Spectra of thirteen planetary nebulae and their nuclei have been published by Swings (rig). Quite a few spectra of southern planetary nebulae have been obtained by Evans with the Radcliffe reflector, but other pressing programmes make progress slow. Evans would be interested to have the advice of other members of the Commission about the best choice of programmes in the southern hemisphere.

Bowen has made extensive measures of long-exposure spectrograms of eight bright planetary nebulae in order to obtain more precise wave-lengths of the forbidden lines. The dispersions ranged from 9 to $57 \AA / \mathrm{mm}$; t the coudé spectrograph of the 200 -inch was used. The wave-lengths of seventy-five forbidden lines have now been determined with an uncertainty of $0.01-0.02 \AA$ for the best-studied lines and $0.2-0.3 \AA$ for the faintest ones. Many of these had uncertainties of I $\AA$ or more in the laboratory predictions.

Great progress has been made in photometry and spectral photometry. At Pretoria 
Koelbloed has made filter photographs for photometry of the distribution of the [O III] lines in southern planetaries. Liller and Aller have made photo-electric photometry of many planetary nebulae, isolating by means of a filter the green nebular lines. They obtained the brightnesses of eighty planetary nebulae and of a number of emission objects in $\mathrm{M}$ 33. In some cases tracings across the nebula and separate measurements of the central stars were made. MacRae and Stock (120) have photo-electrically measured the intensity ratio of the $\mathrm{N}_{1}+\mathrm{N}_{2}$ lines relative to $\mathrm{H} \beta$, using an objective prism. The values, ranging from 8 to 20 , confirm Aller's photographic results. The weak continuum can also be measured. The $\mathrm{N}_{1}+\mathrm{N}_{2}$ lines were found to correspond to 2300 and $\mathrm{I}_{500}$ equivalent angstroms of the continuum in NGC 6826 and 7662 , respectively. Also Aller and Liller have photo-electrically traced the spectra of several bright planetary nebulae. The ratio of $\mathrm{H} \beta$ to the green nebular lines agrees with earlier results, including the photo-electric work of MacRae and Stock. Code has measured photo-electrically the intensities of the lines in NGC 7027.

The project for the measurement of isophotic contours in the planetary nebulae, carried out by Aller, Minkowski and Wilson (IzI), is now completed. The programme includes nineteen objects observed at the coudé focus of the Ioo-inch reflector and sixteen objects observed with the 200-inch. The uniform shell model does not hold in any case. Most planetaries show numerous wisps and condensations, but the Owl nebula, without any visible fine structure, forms a notable exception.

Of great interest is Wilson's(122) work on the kinematics and shapes of planetary nebulae. A great deal of new material, including slitless spectra, slitspectra and multislitspectra, has been accumulated and will be published and discussed in a book by Minkowski and Wilson.

The physical and galactic characteristics of planetary nebulae have been compared by H. M. Johnson (123).

The following studies of individual nebulae have been brought to my attention. Latypov has determined the distance of the ring nebula in Lyra to 570 parsecs from new measurements of the lateral expansion ( 0 "0o \pm 0 o $\left.{ }^{\prime \prime} \mathrm{OOI}\right)$ per year and the known expansion of $24.5 \mathrm{~km}$. $/ \mathrm{sec}$. in the line of sight. Rosino (124) has presented a detailed study of the planetary nebula NGC 7635 . It is suggested that the nebula interacts with interstellar matter. Faint extensions, possibly remnants of old shells or just diffuse emission regions, were discovered by Shajn and Gaze (125) near the planetaries NGC 7293 and 7635 ; such extensions seem to be exceptions rather than a rule. Using Seaton's improved target areas for collisional excitation of forbidden lines and his own spectrophotometric results for NGC 7027, Aller has derived new abundances for this nebula.

\section{Planetary Nebulae: Physics and Dynamics}

Thorough discussions by Aller (126) and Seaton (127) have clarified the situation on the electron temperatures. Both from the cooling effect of the forbidden lines and from the measured intensity ratio of $\lambda 4363$ to the green lines interpreted by means of the new target areas of Seaton(128) Aller concludes that $T_{e}$ is usually higher than $10,000^{\circ}$. In nebulae with faint forbidden lines, such as IC $4 \mathrm{I} 8$, it is about $20,000^{\circ}$. Heating above $25,000^{\circ}$ is excluded because of the collisional excitation of hydrogen. Seaton, using also forbidden lines of other ions, reaches similar conclusions. His paper also contains much of interest about electron densities, abundance ratio $\mathrm{N} / \mathrm{O}$ and the corrections for interstellar reddening. A discussion of the Balmer continuum of IC $4 \mathrm{I} 8$ by Minkowski (r29) confirms the higher temperature.

The successive ionization zones are still subject to new theoretical studies. Van Pelt has made a theoretical study of the shell structure of a planetary nebula treated together with the envelope of the Wolf-Rayet nuclear star. He finds for thirteen nebulae that the shells with successive stages of ionization are indeed as expected. Results for NGC 6543 have been published (130). 
Pikelner (13I) rediscusses the extent of the two ionization zones of $\mathrm{He}$ around an O star. The fact that the Zanstra temperatures derived from He come out higher than those derived from $\mathrm{H}$ is interpreted as due to a real deviation of the nuclear spectrum from a black body and not to incomplete absorption.

Hattori, Kawaguchi, Miyamoto and Saigusa (r32) have computed the intensity ratio He II $4686 / \mathrm{H} \beta$ in terms of the optical thickness of the nebula at the Lyman limit. By means of this expression they derive the optical thickness of a number of nebulae.

The way in which a dark HI region surrounding the luminous part of a planetary nebula may influence the Balmer decrement and the form of the nebula has been investigated by Güssow (133). The empirical relations between Balmer decrement, electron temperature and temperature of the nucleus are newly discussed from this point of view.

Even more complicated is the transfer of the Lyman $\alpha$ radiation, as it depends critically on the state of motion of the nebula and this motion may, in turn, be determined to some extent by the Lyman $\alpha$ radiation. These complications, which had beer. clearly pointed out by Zanstra ( $\mathrm{x}_{34}$ ), also formed a point of discussion at the Cambridge Symposium and have been further treated by the following authors.

Sobolev, continuing his studies of the diffusion of radiation, has solved several problems of importance for the discussion of Lyman $\alpha$ radiation in a nebula or in the interstellar gas. His first study (r35) treats the time-dependent diffusion, thus admitting an estimate of the time during which a Lyman $\alpha$ quantum remains enclosed. His second one (136) treats the coherent scattering and complete redistribution as two limiting cases of a more general theory.

Unno (137) has devoted several studies to the transfer of Lyman $\alpha$ radiation with redistribution inside the line. Successively he compares various approximations to the exact solution, applies it also to HeII radiation and includes natural damping. With Takakubo $\left(\mathrm{r}_{3} 8\right)$ he explains $\mathrm{O}$. C. Wilson's observations by a theory based principally on the radiation pressure on HeII.

Yada (139) has recomputed the transfer of Lyman $\alpha$ radiation, including the effect of 2-photon emission, for nebulae with optical thicknesses of I, 3 and ro in the Lyman continuum. The decrease of Lyman $\alpha$ due to 2 -photon emission is of the order of $\mathrm{I} \%$ if $\tau=$ IO.

With so much activity in the photometric investigation and basic physical theory of the planetary nebulae, it is perhaps somewhat early to try to arrive at an overall dynamical picture of their structure and evolution. Nevertheless a daring attempt in this direction has been made by Gurzadian. First of all, Gurzadian argues that, as the shapes of the planetary nebulae are stable and those of nova shells are not, and as, moreover, there is no known mechanism to decelerate the high velocities, planetary nebulae do not originate from nova shells. He then interprets the anomalous planetary nebulae (of so-called spiral form) as due to local emission of gas streams from a rotating star. Finally, he concludes that the planetary nebulae with a faint extended region around a bright inner region (tentatively called double-volume planetaries) are not the result of successive ejections. The second envelope is formed at a certain evolutionary stage under the influence of the nebula's own Lyman $\alpha$ radiation. The evolution is thought to be:

$$
\text { planetary type } \rightarrow \text { double-volume } \rightarrow \text { ring-shaped. }
$$

A new classification is worked out on the basis of this theory. These studies will be published in a monograph.

H. C. van DE Hulst

President of the Commission 


\section{REFERENCES}

(I) V. S. Safronov, in Problems of Cosmogony, 2.

(2) L. Spitzer, $A P . J$. 120.

(3) V. F. Gaze, C.R. Acad. Sci. U.R.S.S. 95, 95 I, I954; Publ. Crim. Obs. 12, 88, 1954.

(4) B. J. Bok et al., Lg.‡

(5) G. Münch, Publ. Astr. Soc. Pacif. 65, I79, 1953.

(6) H. C. van de Hulst, C. A. Muller and J. H. Oort, B.A.N. no. 452, 1954.

(7) G. Münch, Publ. Astr. Soc. Pacif. 64, 312, 1952.

(8) L. Binnendijk, $A p$. J. I15, 452, I952.

(9) C. S. Beals and J. B. Oke, Mon. Not. R. Astr. Suc. I13, 530, 1953.

(г) T. Adolfsson, Medd. Astr. Obs. Uppsala, nos. Io7 and rog, I954.

(I I) O. Eklöf, Medd. Astr. Obs. Uppsala, no. ro6, 1953.

(12) A. G. P. Velghe, Ann. Astr. Obs. Uppsala, 3, no. 7 .

(13) E. K. Kharadze, Bull. Abastumani Obs. 12, 1952.

(I4) V. B. Nikonov, Bull. Abastumani Obs. I3.

(I5) M. A. Vashakidze, Bull. Abastumani Obs. 14, 1953.

(16) A. F. Torondzhadze and T. A. Kotchlashvili, Astr. J. U.S.S.R. 31, 387, 1954.

(17) A. D. Thackeray, Camb. §

(I8) M. W. Feast, Observatory, 73, 255, I953.

(19) A. E. Whitford, Astr. J. 59, 194, 1954.

(20) G. Schalén, Ann. Astr. Obs. Uppsala, 3, no. 9, 1953.

(2r) S. A. Kaplan and I. A. Klimishin, Astr. J. U.S.S.R. 29, 326, 1952; Lwow Circ. 25, I953.

(22) D. A. Rozhkovski (in the Press).

(23) D. Y. Martynov, Astr. Circ. U.S.S.R. no. I 50, 1954.

(24) E. Schoenberg, Forsch.ber. Obs. Wendelstein, Heft I I.

(25) A. D. Thackeray, Lg.

(26) A. A. Wachmann, Z. Ap. 35, 74, 1954.

(27) L. Divan, Lg.; Ann. d'Astrophys. 17, 456, I954.

(28) O. A. Melnikov, Astr. J. U.S.S.R. 3x, 249, 1954.

(29) P. J. van Rhijn, B.A.N. no. 44I, I953.

(30) J. Borgman, B.A.N. no. 457, 1954.

(3I) H. Kienle, Lg.

(32) G. M. Idliss (in the Press).

(33) H. Zirin, Harvard Bull. no. 921, 1952.

(34) H. C. van de Hulst, Lg.

(35) F. D. Kahn, Mon. Not. R. Astr. Soc. 112, 5 18, 1952.

(36) A. Güttler, $Z$. Ap. 31, r, 1952.

(37) E. Schatzman and R. Cayrel, Lg.

(38) S. Chandrasekhar and E. Fermi, $A p . J .118$, I $13,1953$.

(39) A. A. Hoag, Astr. J. 58, 42, 1953.

(40) W. A. Hiltner, $A p . J$. 120, 41, I78, 367 and 454, 1954.

(4I) A. Elvius, Ann. Stockh. Obs. 17, no. 4, I95I; Lg.

(42) G. A. Shajn, $L g$.

(43) M. T. Martel, $L g$.

(44) M. J. Seaton, $\mathrm{Lg}$.

(45) D. A. Rozhkovski, C.R. Acad. Sci. U.R.S.S. 95, no. I, 1954.

(46) L. Davis, in Vistas in Astronomy; Lg.

(47) T. Gold, Mon. Not. R. Astr. Soc. 112, 215, 1952.

(48) L. Davis, Phys. Rev. I954.

(49) G. R. Burbidge, $A p . J$. 118, 575, 1953.

(50) H. Lambrecht and H. Zimmermann, Lg.; Mitt. Jena, 13; fuller tables in Veröff. Jena.

$\ddagger$ The report of the Liege Symposium on 'Dust in Astronomical Objects', will be referred to as $L g$. $\S$ The report of the Cambridge Symposium on 'Gas Dynamics of Interstellar Clouds' will be referred to as Camb. 
(5I) H. Lambrecht and H. Zimmermann, Mitt. Jena, r3.

(52) S. A. Kaplan, Astr. J. U.S.S.R. 29, 320, I952.

(53) S. A. Kaplan and S. I. Gopasiuk, Lwow Circ. 25, 1953.

(54) G. Herzberg, Lg.

(55) F. D. Kahn, Lg.

(56) The latest paper is L. Spitzer, $A p . J$. III, 593, 1950.

(57) M. Savedoff, Camb.

(58) L. Spitzer, $A p . J$. 120, I, 1954.

(59) P. M. Routly and L. Spitzer, Ap. J. 115, 227, 1952.

(60) L. Spitzer and A. Skumanich, $A p . J$. 116, 452, 1952.

(6r) F. D. Kahn, Lg.

(62) J. H. Oort, B.A.N. no. 455, I954; Camb.

(63) L. Searle, $A p . J$. I16, 650, I952.

(64) A. Schlüter, H. Schmidt and P. Stumpff, $Z$. $A p$. 33, r94, 1953.

(65) G. Münch, $A p . J$. (in the Press).

(66) G. A. Shajn, Astr. J. U.S.S.R. 31, 217, 1954; in Vistas in Astronomy.

(67) E. Schatzman and F. D. Kahn, Camb.

(68) F. D. Kahn, B.A.N. no. 457, 1954 .

(69) S. A. Kaplan, Astr. J. U.S.S.R. 3r, 1954.

(70) E. A. Frieman, $A p . J .120$, 18, 1954.

(7x) S. Chandrasekhar and E. Fermi, $A p . J$. 118, I16, I953; 119, 7, I954.

(72) E. N. Parker, Astr. J. 58, 46, 1953; Ap. J. 117, 169, I953.

(73) G. C. McVittie, $A p . J$. 119, 352, r954.

(74) E. Fermi, $A p . J$. 119, I, I954.

(75) S. A. Kaplan and W. I. Pronik, Astr. J. U.S.S.R. 32, no. 2, 1955.

(76) S. A. Kaplan and W. I. Pronik, J. Exp. Theor. Phys. (in the Press).

(77) S. A. Kaplan, Astr. J. U.S.S.R. 29, 326, 1952.

(78) A. I. Lebedinsky, C.R. Acad. Sci.U.R.S.S. 84, no. 2 , 1952.

(79) M. Schwarzschild and L. Spitzer, $A p . J .114,385$, r95 ; 118, 106, 1953.

(8o) K. Takakubo, Sci. Rep. Tohuku Univ. 37, 320, 1953.

(8I) D. ter Haar, $Z . A p \cdot$ 32, 25I, I953.

(82) S. B. Pikelner, C.R. Acad. Sci. U.R.S.S. 88, 229, I953; Publ. Crim. Obs. 10, 74, 1953.

(83) J. H. Oort, B.A.N. no. 455, r954; Camb.

(84) G. A. Shajn and V. F. Gaze, Publ. Crim. Obs. ro, 152, 1953; Astr. J. U.S.S.R. 31, 305, 1954.

(85) S. Sharpless, $A p . J .119,334$, I954.

(86) G. A. Shajn et al., Publ. Crim. Obs. 8, 3, 1952; C.R. Acad. Sci. U.R.S.S. 82, 857, 1952; Astr. J. U.S.S.R. 30, $125, \mathrm{r} 30,1953$.

(87) G. A. Shajn et al., C.R. Acad. Sci.U.R.S.S. 90, 74I, 1953; Publ. Crim. Obs. 11, 22, I954.

(88) G. A. Shajn et al., Astr. J. U.S.S.R. 30, 135, 1953.

$(89$ C. S. Gum and G. de Vaucouleurs, Observatory, 73, I52, I953.

(90) V. G. Fesenkov and D. A. Rozhkovski, Astr. J. U.S.S.R. 30, no. I, 1953; 31, no. I, r954; 3I, no. 4, I954.

(9I) M. Savedoff, Camb.

(92) B. J. Bok, Camb.

(93) B. J. Bok, Lg.

(94) G. A. Shajn, V. F. Gaze and S. B. Pikelner, Publ. Crim. Obs. 12, 64, 1954; Astr. J. U.S.S.R. 31, 105, 1954; Lg.

(95) H. M. Johnson, $A p . J$. 118, 370, 1953.

(96) H. M. Johnson, $A$ p. J. I17, 235, 1953.

(97) P. Wellmann, $A p . J$. 116, 452, 1952.

(98) See, for example, four papers by Baade, Minkowski, Aller and Greenstein in $A p . J$. r19, no. I, I954, and several papers by Shklovsky in recent issues of Astr. J. U.S.S.R.

(99) W. W. Morgan and A. Blaauw, Ap. J. 119, 625, I954.

(I0o) G. Courtès, Camb.; Lg. 
(IoI) V. G. Fesenkov, D. A. Rozhkovski et al., C.R. Acad. Sci. U.R.S.S. 88, no. 3, 1953.

(I02) V. G. Fesenkov, D. A. Rozhkovski et al., Astr. J. U.S.S.R. 31, no. 3, I954.

(103) S. B. Pikelner, Publ. Crim. Obs. 12, 93, 1954.

(I04) J. W. Chamberlain, $A p . J .117,3^{87}$ and 399, 1953.

(105) R. Minkowski, Camb.

(I06) M. Johnson, Mon. Not. R. Astr. Soc. 113, 468, 1953.

(I07) C. S. Gum, Observatory, 72, I 5 I, 1952; 73, 123, 1953.

(Iо8) L. Biermann, Camb.

(109) L. E. Gurevich, in Problems of Cosmogony, 3, 94 (Moscow, 1954).

(I Io) H. Bondi, Mon. Not. R. Astr. Soc. I12, I95, 1952.

(I I I) E. Schatzman, Camb.

(I 12) K. N. Dodd and W. H. McCrea, Mon. Not. R. Astr. Soc. I12, 205 and 374, I952; I13, 162 and $484,1953$.

(II3) G. Herbig et al., J.R. Astr. Soc. Can. 46, 222, 1952.

(I I 4) K. G. Henize, $A p$. $J$. I19, 459, I954.

(II5) G. Haro et al., Bol. Obs. Tonantz. Tacubaya, nos. 7 and 8, 1953.

(II6) G. H. Herbig, $A p . J$. I13, 697, r95I.

(II7) G. Haro, $A p . J .1 \times 5,572$, I952.

(1 18) A. I. Lebedinsky, C.R. Acad.Sci. U.R.S.S. 84, no. 3, 1952; 92, no. 3, I953.

(I I9) P. Swings, Ann. Astrophys. I5, 290, I952.

(I 20) D. A. MacRae and J. Stock, Nature, Lond., I73, 589, I954.

(12I) L. H. Aller, R. Minkowski and O. C. Wilson, $A p$. J. I20, 26I, I954.

(122) O. C. Wilson, $A p . J .117,264$, I953.

(123) H. M. Johnson, $A p . J$. 120, 182, I954.

(124) L. Rosino, Publ. Oss. Bologna, 6, no. 3, 1953.

(125) G. A. Shajn and V. M. Gaze, Publ. Crim. Obs. 11, 18, 1954.

(126) L. H. Aller, $A p$. $J$. 118, 547, I953.

(127) M. J. Seaton, Mon. Not. R. Astr. Soc. I14, I54, I954.

(128) M. J. Seaton, Proc. Roy. Soc. A, 218, 400, 1953.

(129) R. Minkowski, Publ. Astr. Soc. Pacif. 65, 16I, I953.

(130) A. van Pelt, Proc. Kon. Ned. Akad. Wet. 56, 67, 1953 (Amsterdam Circ. no. 7).

(131) S. B. Pikelner, Publ. Crim. Obs. 10, 183, r953.

(132) T. Hattori et al., Proc. Astr. Soc. Japan, 4, I52, 1953.

(133) K. Güssow, to appear in $Z$. $A p$.

(134) H. Zanstra, B.A.N. no. 40r, I949.

(135) V. V. Sobolev, Astr. J. U.S.S.R. nos. 4 and 5, 1952.

(136) V. V. Sobolev, Bull. Leningrad Obs. (in the Press).

(137) W. Unno, Proc. Astr. Soc. Japan, 3, I58, 1952; 3, I78, 1952; 4, 100, 1952.

(138) W. Unno and K. Takakubo, Proc. Astr. Soc. Japan, 3, 195, 1952.

(139) B. Yada, Proc. Astr. Soc. Japan, 5, 128, 1953; 6, 67, 1954.

\section{4a. SUB-COMMISSION FOR THE CATALOGUE OF DIFFUSE EMISSION NEBULAE}

\section{Desirability of a new catalogue}

The use of cameras with a high aperture ratio and a wide field, combined with the possibility to isolate by means of very sensitive plates and red filters (sometimes multilayer interference filters) a narrow wave-length interval around $\mathrm{H} \alpha$ has enormously increased the possibilities for direct photography of emission nebulae. Many new emission nebulae have been discovered in the last few years. In addition, very faint emission was found to be present nearly everywhere at low galactic latitudes. The brighter nebulosities, among which are the emission nebulae in the usual sense as the brightest or most striking parts, are so crowded and interconnected in some regions of the sky that a division into 